\title{
Avaliaçäo crítica do SeVElamer no tratamento dA HIPERFOSFATEMIA EM PACIENTES COM INSUFICIÊNCIA RENAL CRÔNICA
}

\author{
Ricardo Sesso*, Marcos B. Ferraz \\ Trabalho realizado no Grupo Interdepartamental de Epidemiologia Clínica/Centro Paulista de \\ Economia de Saúde e Disciplina de Nefrologia, Escola Paulista de Medicina, Unifesp, S. Paulo, SP
}

RESUMO - OвJetivo. Avaliar em pacientes com insuficiência renal crônica (IRC), a efetividade e os custos do sevelamer, um polímero catiônico livre de alumínio e cálcio, que é um novo quelante de fósforo no trato gastrointestinal.

Métodos. Revisão da literatura e avaliação crítica de seis ensaios clínicos publicados sobre efetividade e duas avaliações econômicas do sevelamer em pacientes com IRC.

Resultados. O sevelamer é um quelante de fosfato efetivo (utilizado na dose média de 3,5g/dia dividido em 3 doses às refeições) e com efeito similar ao obtido com sais de cálcio, além de não apresentar os efeitos colaterais destes últimos (elevação do produto cálcio $\mathrm{x}$ fósforo, hipercalcemia, calcificação vascular, musculo- esquelética e cardíaca, etc.). Além disso, o sevelamer promove redução em cerca de $30 \%$ nos níveis de LDL colesterol. Custos diretos do sevelamer são maiores que os dos sais de cálcio, embora os custos totais possam ser menores devido a menor taxa de complicações clínicas e hospitalizações.

CONCLUSÕES. Sevelamer agrega valor terapêutico importante em pacientes com IRC e hiperfosfatemia. Estudos fármaco-econômicos devem ser realizados em nosso meio para se avaliar a relação custoefetividade do uso do sevelamer.

UnITERMOS: Sevelamer. Hiperfosfatemia. Insuficiência Renal Crônica. HiperparatireoidismoSecundário.

\section{INTRODUÇÃO}

Em indivíduos normais, a concentração sérica de fósforo é mantida numa faixa estreita apesar do consumo variável de fósforo na dieta. O conteúdo médio diário de fósforo numa dieta ocidental é entre $\mathrm{I}$ a $\mid, 8 \mathrm{~g}^{\mid}$. Desta quantia, $70 \%$ são absorvidos pelo trato gastrointestinal e excretados pelos rins. $\mathrm{Na}$ insuficiência renal crônica (IRC), o sistema para manutenção de balanço do fósforo é rompido pela perda de nefrons. Conforme o ritmo de filtração glomerular (RFG) de fósforo cai, há uma adaptação renal caracterizada por um declínio na reabsorção tubular de fósforo, causando fosfatúria aumentada nos nefrons residuais. Além de certo ponto (RFG $<25 \mathrm{ml} /$ min.), a elevação nos níveis de paratohormônio (PTH) não consegue aumentar mais a fosfatúria, e ocorre hiperfosfatemia ${ }^{1,2}$. 0 hiperparatireoidismo secundário persistente pode causar doença óssea caracterizada pela osteíte fibrosa cística. O hiperparatireoidismo secundário é uma complicação comum da IRC, evidente com modestas reduções no

\footnotetext{
* Correspondência:

Rua Botucatu, 740-CEP: 04023-900-São Paulo-SP Fone:(1 1) 55746300-Fax:(1 1)55739652 E-mail:rsesso@nefro.epm.br
}

$\mathrm{RFG}^{3}$. Se não for tratado, o hiperparatireoidismo secundário pode levar a significante morbidade devido a dor, risco aumentado de fraturas, deformidades ósseas e anemia ${ }^{4,5}$, e tem sido postulada sua contribuição para hipertensão, doença vascular, prurido e disfunção sexual|-6.

A redução da absorção do fósforo é crucial para prevenir a hiperfosfatemia e o hiperparatireoidismo que se desenvolvem em pacientes com número diminuído de nefrons funcionais. Como o fósforo é absorvido a partir da dieta, pacientes com IRC avançada são colocados em dietas com restrição de fósforo. Entretanto, esta redução de fosfato na dieta é usualmente insuficiente e a maioria dos pacientes usa quelantes de fosfato'. Alimentos ricos em fósforo incluem derivados de leite, proteínas animais (carnes), legumes, grãos e chocolate. A maioria dos nutricionistas recomenda restrição de fósforo na dieta para menos de $0,8-1,0$ $\mathrm{g} /$ dia para a maioria dos pacientes com insuficiência renal crônica terminal (IRCT). Apesar dessa restrição, o fósforo absorvido excede a quantidade de fósforo removida pela diálise. Os benefícios da restrição de fosfato devem ser balanceados com o potencial risco de desnutrição proteico-calórica. Num estudo norte-americano em pacientes com IRCT em hemodiálise entre 1990-93 $(\mathrm{N}=6407)^{7}$, ob- servou-se que $60 \%$ deles tinham níveis de fósforo sérico $>5,5 \mathrm{mg} / \mathrm{dl} \mathrm{e} 39 \%$ tinham níveis $>6,5 \mathrm{mg} / \mathrm{dl}$. Pacientes com níveis acima desse valor tinham um excesso de risco de morte (risco relativo $[R R]=1,27$ ) comparado àqueles com fósforo sérico entre 2,4 e $6,5 \mathrm{mg} / \mathrm{dl}$. 0 mecanismo pelo qual o fósforo sérico elevado resulta em mortalidade pode estar relacionado a um elevado produto $\mathrm{Ca} \times \mathrm{PO}_{4}$, mas não é explicado por níveis de PTH. Produto $\mathrm{Ca} x$ $\mathrm{PO}_{4}$ acima de $72 \mathrm{mg}^{2} / \mathrm{dl}^{2}$ foi observado em $20 \%$ dos pacientes e esteve associado a um significante aumento no risco de morte $(R R=I, 34)$ comparado àqueles com valores entre $42 \mathrm{e} 52 \mathrm{mg}^{2} / \mathrm{dl}^{27}$. Portanto, um vigoroso controle do fósforo em níveis abaixo de 6,5 $\mathrm{mg} / \mathrm{dl}$ é de vital importância para pacientes em diálise crônica, o que deve ser alcançado não somente com o regime dialítico, mas primariamente pela manipulação da absorção do fósforo no trato gastrointestinal.

A sobrevida de pacientes com IRCT é substancialmente reduzida, e a doença cardiovascular é responsável por aproximadamente $50 \%$ das mortes entre os pacientes em diálise ${ }^{8,9}$. Fatores contribuintes incluem hipertensão, intolerância a glicose, dislipidemia, elevação de homocisteina sérica, e anormalidades no metabolismo do cálcio e fósforo ${ }^{10-13}$. A associação da elevação do produto $\mathrm{CaxPedo}$ 
fósforo sérico com mortalidade aumentada pode ser secundária a calcificação de artérias coronárias com subseqüente doença cardíaca isquêmica, infarto do miocárdio e insuficiência cardíaca, ${ }^{7}, 14,15$.

Sais de alumínio são quelantes de fosfato eficazes, sendo que o hidróxido de alumínio foi o primeiro composto oral amplamente utilizado há mais de 30 anos. Mas o alumínio acumula nos tecidos de pacientes com insuficiência renal e está associado com significante toxicidade ${ }^{16}$. O uso crônico de sais de alumínio resulta em anemia hipocrômica microcítica, osteomalácia, miopatia e raramente demência ${ }^{17,18}$.

Sais de cálcio também são quelantes efetivos de fosfato e se tornaram os principais produtos usados com esta finalidade durante a última década. Entretanto, uma porcentagem do cálcio ingerido éabsorvida, causando maior risco de calcificação metastática em alguns pacientes $^{19,20}$. Apesar de sua eficácia, baixo custo, garantia de suplementação de cálcio nos pacientes, ele possui desvantagens: a) depende de pH gástrico para dissolução e eficácia, b) liga-se ao ferro oral, habitualmente prescrito a pacientes renais crônicos, tornando os dois menos efetivos $\left.{ }^{21}, c\right)$ freqüência relativamente alta de hipercalcemia com resultante elevação do produto $\mathrm{Ca} \times \mathrm{P}$.

Lesões ateroscleróticas calcificadas, particularmente em artérias coronarianas, são comuns e progressivas em pacientes com IRCT ${ }^{22,23}$. Calcificações coronarianas documentadas por tomografia computadorizada com raios de elétrons foram detectadas em homens e mulheres com menos de 30 anos de idade e em programa de diálise por mais de cinco anos $^{23}$. Calcificações de valvas cardíacas também foram documentadas nesses pacientes ${ }^{24}$. A combinação de aporte excessivo de cálcio e elevação de níveis de fósforo em pacientes com IRCT causa aumento no produto $\mathrm{Ca} \times \mathrm{P}$, que pode favorecer a deposição de depósitos calcificados em tecidos moles ${ }^{7,14}$. Há várias fontes de aporte excessivo de cálcio em pacientes em diálise: dieta, solução de diálise, quelantes de fósforo com base de cálcio e terapia com vitamina $\mathrm{D}^{25}$. Tem sido recomendado que se mantenha controle estrito do fósforo sérico em níveis entre 2,5 e 5,5 mg/dl edo produto CaxPabaixo de $55 \mathrm{mg}^{2} / \mathrm{dl}^{2}$, para reduzir o risco de calcificação urêmica, doença cardiovascular e morte cardíaca ${ }^{7,14}$. Além dis- so, recomenda-se utilizar banhos de diálise pobres em cálcio ${ }^{25}$, análogos de vitamina $D$ que não aumentem a absorção intestinal de cálcio e fósforo, e de quelantes de fósforo livres de cálcio ${ }^{7,14}$. Há estudos indicando que as calcificações arteriais aumentam com o uso de quelantes de fosfato a base de cálcio ${ }^{26}$. Portanto, é necessária a disponibilidade de quelantes de fosfato efetivos, sem os efeitos colaterais do alumínio ou do cálcio.

\section{Sevelamer}

Sevelamer $\left(\right.$ Renagel $\left.{ }^{R}\right)$ é um polímero catiônico (polialilamina-hidroclorada) livre de alumínio e cálcio e que se liga ao fosfato ${ }^{27}$. Ele é ligado por múltiplas aminas espaçadas por moléculas de carbono. Estas aminas se tornam parcialmente carregadas de prótons no intestino e interagem com moléculas de ânion fosfato através da carga e da ligação com hidrogênio. In vitro, I g de sevelamer liga $5 \mathrm{mmol}$ de fosfato em $\mathrm{pH}$ 7. Sevelamer liga-se preferencialmente a ânions trivalentes, como fosfato e citrato. Sevelamer também se liga a ácidos biliares e aminoácidos conjugados carregados negativamente, os quais, como o fosfato, são abundantes no intestino durante as refeições. Aligação do sevelamer comácidos biliares leva ao aumento da excreção fecal de ácidos biliares e abaixa o LDL colesterol. Este decréscimo no LDL colesterol é a propriedade farmacológica primária do sevelamer. Estudos em animais e em humanos demonstraram que o sevelamer não é absorvido.

\section{Efetividade do sevelamer em pacientes em diálise (Tabela I)}

Para se verificar a efetividade, segurança e tolerância do sevelamer, 24 indivíduos voluntários sadios foram estudados ${ }^{27}$. Indivíduos receberam sevelamer I, 2,5 e $5 \mathrm{~g}$ ou placebo três vezes ao dia imediatamente antes das refeições por sete dias. Houve um declínio na excreção de fósforo urinário, sendo o conteúdo médio por dia de $870 \mathrm{mg}$ no grupo placebo, $762 \mathrm{mg}, 625 \mathrm{mg}$ e $530 \mathrm{mg} /$ dia nos grupos sevelamer $\mathrm{I}, 2,5$ e $5 \mathrm{~g} / 3 x \mathrm{xdia}(\mathrm{p}<0,00 \mathrm{I})$. 0 conteúdo fecal de fósforo foi marcadamente maior no grupo que recebeu $5 \mathrm{~g}$ vs. placebo. Sevelamer também diminuiu o colesterol total em $27,5 \mathrm{mg} / \mathrm{dl}, 21,3 \mathrm{mg} / \mathrm{dl}$ e $41,8 \mathrm{mg} / \mathrm{dl}$ nos grupos I, 2,5 e $5 \mathrm{~g}$.

O estudo inicial de fase II em pacientes com IRCT foi um ensaio clínico randomizado, controlado com placebo, de grupos paralelos, duplo cego em 36 pacientes em hemodiálise ${ }^{28}$. A redução de fósforo sérico foi significativamente maior após tratamento por duas semanas com sevelamer $(6,6 \pm 2,1 \mathrm{mg} / \mathrm{dl}$ para $5,4 \pm 1,5 \mathrm{mg} / \mathrm{dl}, \mathrm{p}=0,037)$ comparado com placebo $(7,0+2,1 \mathrm{mg} / \mathrm{dl}$ para $7,2 \pm 2,4 \mathrm{mg} / \mathrm{dl})$. Colesterol sérico total e a fração LDL estiveram significativamente reduzidos nos pacientes tratados com sevelamer.

Um estudo aberto de titulação de dose examinou a eficácia do sevelamer em pacientes com IRCT ${ }^{29}$. Após período de duas semanas sem uso de quelantes, sevelamer foi iniciado em 48 pacientes em doses de $0,5, I, 0$ ou $1,5 \mathrm{~g} 3 \mathrm{x} / \mathrm{d}$ dado às refeições por oito semanas. Com sevelamer ofósforo declinou e retornou aos níveis pré-'washout' após quatro semanas. PTHi caiu de 395 para 283 pg/mol após seis semanas, apesar do cálcio sérico estar persistentemente mais baixo.

Um estudo randomizado aberto foi feito para se comparar o efeito do sevelamer com ou sem aporte noturno de carbonato de cálcio no nível sérico de fósforo e PTH de pacientes renais crônicos ${ }^{30}$. Após 12 semanas de uso de medicação, sevelamer, sem ou com cálcio, foi igualmente efetivo na redução de fósforo (redução média $-2,4 \mathrm{mg} / \mathrm{dl}$ vs. $-2,3 \mathrm{mg} / \mathrm{dl}$ ). Sevelamer com cálcio foi associado a pequeno aumento não significante no cálcio sérico. Durante a fase de tratamento, a redução no PTH tendeu a ser maior no grupo sevelamer-cálcio (diferença mediana -67 vs. -23 pg/ml no grupo sevelamer, $p=0,07$ ).

Um estudo aberto, randomizado, cruzado, foi realizado para avaliar a efetividade e a segurança do sevelamer em controlar a hiperfosfatemia em pacientes com IRCT ${ }^{31}$. Pacientes receberam sevelamer ou acetato de cálcio durante oito semanas. Houve um decréscimo similar nos valores de fósforo sérico durante o estudo no grupo sevelamer $(-2,0 \pm 2,3 \mathrm{mg} / \mathrm{dl})$ e acetato de cálcio $(-2, I \pm I, 9 \mathrm{mg} / \mathrm{dl})$. Vinte e dois por cento dos pacientes desenvolveram hipercalcemia no grupo que recebeu acetato de cálcio vs. $5 \%$ no grupo sevelamer. Pacientes tratados com sevelamer apresentaram um decréscimo de $27 \%$ nos níveis de LDL.

Em outro estudo, 172 pacientes em hemodiálise foram avaliados prée pós o uso do sevelamer ${ }^{32}$. Houve queda significativa do fósforo sérico após oito semanas de uso de 


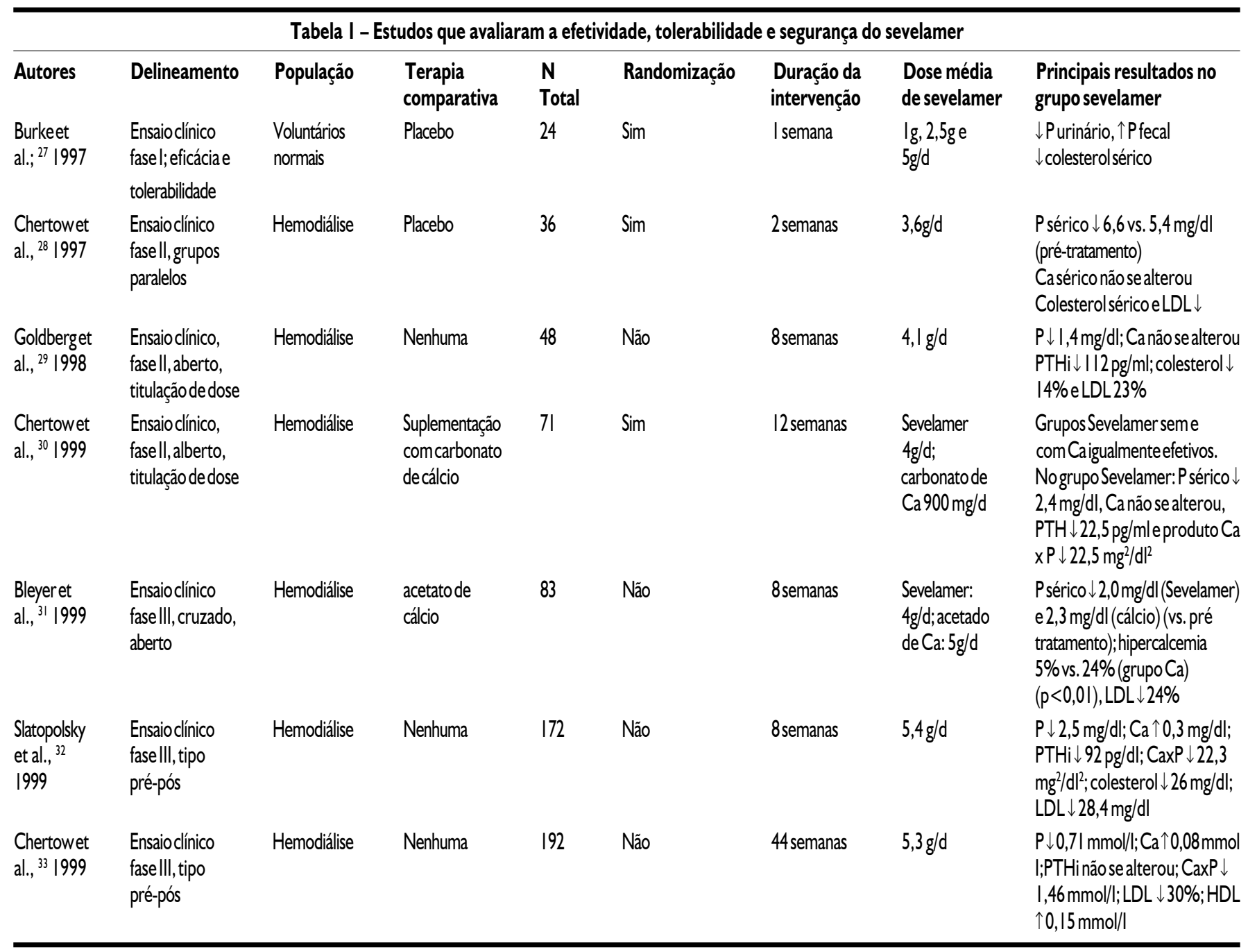

sevelamer, em média, de $9, I \pm 2,4 \mathrm{mg} / \mathrm{dl}$ para $6,6 \pm 1,9 \mathrm{mg} / \mathrm{dl}(\mathrm{p}<0,000 \mathrm{I})$. O cálcio sérico aumentou de $9, I \pm 0,8$ para $9,4 \pm 0,9$ e $\mathrm{PTHi}$ mediano declinou de 316 para $224 \mathrm{pg} / \mathrm{ml}$ $(p<0,000 \mathrm{I})$. Além disso, sevelamer reduziuo colesterol total sérico médio de $|7|, 0 \pm 43, \mid$ para I45,0 $038,7 \mathrm{mg} / \mathrm{dl}(\mathrm{p}<0,000 \mathrm{I})$ e a LDL média de $102,0 \pm 34,9$ para $75,6+29,4 \mathrm{mg} / \mathrm{dl}$ $(\mathrm{p}<0,000 \mathrm{I})$.

A efetividade a longo prazo (46 semanas) do sevelamer foi avaliada em um ensaio clínico aberto em I 23 pacientes adultos em hemodiálise ${ }^{33}$. O estudo foi do tipo 'antes-depois' $\mathrm{e}$ não teve grupo controle placebo. Ao final do tratamento, o fósforo sérico diminuiu em média $2,20 \pm 2,39 \mathrm{mg} / \mathrm{dl}$, o cálcio aumentou $0,32 \pm 0,88 \mathrm{mg} / \mathrm{dl}$, o produto $\mathrm{Ca} \times \mathrm{P}$ diminuiu $-I, 46 \pm I, 78 \mathrm{mmol} / \mathrm{I}(\mathrm{p}<0,000 \mathrm{I}$ para todas as comparações). Não houve alteração no nível de cálcio ao final do período de 'washout' e ao final do tratamento. Níveis séricos de LDL caíram em média $0,81 \pm 0,75 \mathrm{mmol} / \mathrm{I}(-30 \%$, $\mathrm{p}<0.000 \mathrm{I})$ e $\mathrm{HDL}$ colesterol aumentou em média $18 \%(p<0,000 \mathrm{I})$.

\section{Discussão sobre estudos de efetividade do sevelamer}

Os ensaios clínicos realizados indicam que o sevelamer é uma alternativa segura e efetiva em relação ao uso de cálcio oral para o manuseio da hiperfosfatemia na IRCT. A dose média de sevelamer usada foi de $1,2 \mathrm{~g}$ $3 x / d$ (variação de $0,8 \mathrm{~g}$ a $1,6 \mathrm{~g} 3 \mathrm{x} / \mathrm{d}$ ). Sevelamer diminuiu o fosfato sérico numa variação média entre $-0,45$ e $-0,81 \mathrm{mmol} / \mathrm{l}$. Em geral, um pequeno aumento do cálcio sérico foi observado com o uso isolado de sevelamer, embora com menor risco de hipercalcemia quando comparado ao uso de acetato de cálcio. Sevelamer foi menos efeti- vo que o acetato de cálcio na supressão do PTHi, e em diversos casos deve ser usado em combinação com vitamina $D$ e/ou suplementação de cálcio. Houve um decréscimo contínuo e significante do produto $\mathrm{Ca}$ X. P. A longo prazo houve um decréscimo de LDL colesterol de $30 \%$ e aumento no HDL em $18 \%$.

Sevelamer foi bem tolerado, apresentando poucos eventos adversos e similares aos dos grupos placebo. No entanto, a maioria desses estudos é de curto período de intervenção. Apenas um teve a duração de cerca de um ano de seguimento e, mesmo assim, este último não foi randomizado, mascarado, não teve grupo controle concorrente e houve perda de seguimento de número substancial de pacientes. Algumas potenciais vantagens do uso do sevelamer são as seguintes: I) adia a necessidade ou evita a exposição ao alumínio, às vezes requerido para o tratamento de 
hiperfosfatemia refratária; 2) permite o início ou a intensificação do uso de calcitriol ou outro análogo da vitamina $D$, sem risco de hipercalcemia; 3) a redução de dose ou parada do uso de sais de cálcio pode evitar as complicações da terapia com cálcio; 4) redução na concentração de colesterol sérico. Alternativamente, a combinação de sevelamer + cálcio pode ser ótima, particularmenteem pacientes com ingestão baixa de cálcio na dieta.

Aterosclerose é acelerada em até 20 anos em pacientes em diálise ${ }^{34}$ comparado a população geral, e doença cardiovascular é responsável pela morte de $50 \%$ destes indivíduos. Como o sevelamer diminui o fósforo sérico com pouco ou nenhum efeito sobre o cálcio, isto resulta num decréscimo do produto $\mathrm{Cax}$ $\mathrm{P}$. $\mathrm{O}$ aumento no produto $\mathrm{Ca} \times \mathrm{P}$ tem sido associado com calcificação aórtica aumentada em ratos urêmicos ${ }^{35} \mathrm{e}$ em pacientes em hemodiálise $e^{34,35}$. O uso do sevelamer pode potencialmente diminuir a precipitação de cálcio e a calcificação na vasculatura. A redução do produto $\mathrm{Ca} \times \mathrm{P}$ está entre os benefícios mais importantes do sevelamer, pois está demonstrado que elevação neste produto aumenta o risco de morte nos pacientes ${ }^{7}$. Num estudo recente foi mostrado que o sevelamer atenua a progressão de calcificação coronariana e aórtica comparado a quelantes de fosfato à base de cálcio ${ }^{36}$. Outro estudo prospectivo com três anos de seguimento está em curso nos EUA comparando-se a mortalidade e a morbidade de 2000 pacientes em diálise recebendo sevelamer ou quelantes de fosfato à base de cálcio.

Níveis de colesterol se associam com risco aumentado de morte em pacientes em hemodiálise ${ }^{37}$, particularmente de causa cardiovascular. Os benefícios relativos da modificação do perfil lipídico nos pacientes com IRC ainda são desconhecidos. Concentrações elevadas de colesterol sérico foram preditivas de óbito em pacientes com IRCT e diabetes ${ }^{38}$. Anormalidades de lipídios em pacientes em diálise podem contribuir para o risco aumentado de aterosclerose coronariana e em outros leitos vasculares ${ }^{39}$. A redução de cerca de $30 \%$ no LDL colesterol promovido pelo sevelamer pode potencialmente diminuir a aterosclerose nos pacientes em diálise. A utilidade do efeito redutor de lipídios do sevelamer precisa ser determinada em estudos prospectivos.
Uma preocupação importante com agentes quese ligam ao fosfato éque eles interfiram com a absorção de outras drogas. Sais de cálcio podem interferir com a absorção de aspirina, digoxina, ferro, isoniazida equinolonas, enquanto os sais de alumínio interferem comaabsorção de aspirina e quinolonas. Foi demonstrado que o sevelamer não interfere com a absorção de enalapril ou metoprolol, dois agentes antihipertensivos comumente usados ${ }^{40}$.

\section{Avaliações econômicas do sevelamer em pacientes em com insuficiência renal crônica}

Há apenas dois artigos publicados na literatura científica sobre análise econômica do sevelamer. No primeiro foi testada a hipótese de que pacientes tratados com sevelamer possam ter menor taxa de hospitalização comparado a pacientes recebendo quelantes de fósforo contendo cálcio ${ }^{41}$. Este estudo foi realizado nos Estados Unidos e consideraram-se as tabelas de reembolso pagas pelo Medicare. 0 delineamento foi do tipo caso-controle retrospectivo com 152 pacientes tratados com sevelamer e 152 controles das mesmas unidades de diálise. Pacientes do grupo sevelamer haviam participado de um estudo de longa duração para avaliar segurança e eficácia da medicação; o grupo controle foi posteriormente selecionado ao acaso nas mesmas unidades de diálise de onde os casos vieram. A dose média de sevelamer foi de $5,3 \mathrm{~g} / \mathrm{d}$ e 0 período médio de tratamento foi de 17 meses. Apesar do pareamento entre casos e controles para idade, sexo, raça, diabetes e localização geográfica, as taxas de história de acidente cerebrovascular e de doença gastrointestinal com sangramento, e as taxas prévias de hospitalização foram maiores no grupo controle. Em análise multivariada, o risco de hospitalização foi $46 \%-54 \%$ menor no grupo sevelamer $(p=0,03)$. Neste grupo, o índice de hospitalização devido ao acesso vascular e a causa cardíaca foi reduzido em $30 \%-40 \%$ (p ns). Gastos cobertos pelo Medicare por paciente por mês para os grupos controle e sevelamer foram, respectivamente, hospitalares US\$ 1992 e US\$ 1149, ambulatoriais US\$248I e US\$2423, honorários médicos US\$ 748 e US\$ 537 e, incluindo-se os demais custos, o total geral foi de US\$5866 e US\$ 4422. Portanto, os gastos foram quase US\$ 1400 a menos por paciente por mês no grupo sevelamer, eqüivalendo a economia acima de US\$ 16.500 por paciente por ano. Este estudo apresenta algumas limitações: I) tem delineamento retrospectivo; 2) observou-se diferenças de número de comorbidades entre os grupos; 3) a participação num estudo anterior dos pacientes do grupo sevelamer pode ter implicado em melhor acompanhamento médico. Ainda assim, análise multivariada manteve o menor risco (cerca de 50\%) de primeira hospitalização no grupo sevelamer. Isto ocorreu devido, talvez, a menor hospitalização relacionada ao acesso vascular e causa cardiovascular. Risco de mortalidade também foi menor no grupo sevelamer (RR: 0,56, intervalo de confiança $95 \%: 0,25-1,29$, p ns). É necessário a realização de estudos prospectivos, preferencialmente ensaios clínicos randomizados com número de pacientes que proporcione poder estatístico suficiente para as comparações desejadas. Outras limitações deste estudo referentes aos aspectos de análise econômica são as seguintes. Custos indiretos (ausência de trabalho, morte) não foram computados; análise de custo-efetividade (custo por ano adicional de vida) não foi feita; análise de incremento comparando-se o custo adicional por ano de vida ganho com o tratamento alternativo não foi realizado; análise de sensibilidade (variando-se algumas pressuposições e repetindo-se as análises) não foi feita; 17 meses de seguimento talvez seja pouco para se avaliar efeitos cardiovasculares, osteopatia e mortalidade, e desfechos relevantes relacionados a doença cardiovascular e osteopatia não foram medidos. Os resultados sugerem benefícios econômicos com o uso do sevelamer nessa população, no entanto, precisam ser confirmados em estudos locais.

Brophy et al. ${ }^{42}$ realizaram um estudo de custo-efetividade comparando sevelamer e carbonato de cálcio associado a atorvastatina para redução de LDL em pacientes com IRC. Foi construído um modelo de análise de decisão baseado num paciente com IRC com moderada hiperfosfatemia $(6$ a $7,5 \mathrm{mg} / \mathrm{dl}) \mathrm{e}$ LDL plasmático entre 131 e $160 \mathrm{mg} / \mathrm{dl}$, com o objetivo de se atingir redução de $35 \%$ no LDL. Foram calculados os custos por um ano das medicações e exames laboratoriais. A razão de custo-efetividade (custo por paciente atingindo o nível de LDL objetivado) foi marcadamente favorável ao uso de carbonato de cálcio+atorvastatina vs. sevelamer 
(US\$ I 387 vs. US\$ 3|45). Análise de sensibilidade continuou favorecendo o uso de cálcio + atorvastatina mesmo considerando redução de custo de $50 \%$ do sevelamer (US\$ 1359 vs. US\$ 1759). Entretanto, o estudo apresenta diversas deficiências. I) 0 modelo de análise avaliou pacientes com IRC + hiperfosfatemia e hiperlipidemia concorrentes, objetivando a redução do LDL, e é mais provável que na prática o sevelamer seja usado para tratar pacientes com hiperfosfatemia em hemodiálise; 2) os custos de reações adversas não foram incluídos; 3 ) os dados coletados para sevelamer e atorvastatina foram de populações diferentes de pacientes e avaliados em diferentes pontos do tempo; 4) $O$ modelo não abordou o desenvolvimento de hipercalcemia.

\section{Dados nacionais}

No Brasil, estima-se que em dezembro de 200 I havia 57.000 pacientes com IRCT em tratamento dialítico e, destes, ao menos 90\%-95\% usando quelantes de fosfato. É possível que todos os pacientes usando $\mathrm{Al}(\mathrm{OH})_{3}$ (estima-se entre $5 \%$ e $7 \%$ ) mudem para sevelamer. Dos pacientes que tomam sais de cálcio, pelo menos $25 \%$ permanecem com hiperfosfatemia e deveriam trocar rapidamente para sevelamer. A porcentagem de pacientes recebendo sais de cálcio que passem a usar sevelamer pode atingir $50 \%$ após cinco anos.

\section{Conclusão}

Sevelamer agrega valor terapêutico importante para pacientes com IRCT com hiperfosfatemia que não conseguem tolerar ou que apresentem efeitos colaterais decorrentes do uso de sais de cálcio ou alumínio. Há indícios de que, a longo prazo, o uso do sevelamer comparado a outros quelantes traga benefícios na redução da morbidade e mortalidade cardiovascular. $\mathrm{O}$ aumento dos custos diretos decorrentes do uso do sevelamer poderá resultar em economia quando consideramos os custos devido a morbidade e hospitalizações evitadas. Estudos fármaco-econômicos precisam ser realizados em nosso meio para melhor avaliarmos a relação custo-benefício do uso do sevelamer, definirmos o custo adicional por ganho adicional na saúde dos pacientes, permitindo assim termos mais subsídios para a utilização desta medicação em nosso meio.

\section{SUMMARY}

Critical appraisal of sevelamer for THE TREATMENT OF HYPERPHOSPHATEMIA IN PATIENTS WITH CHRONIC RENAL FAILURE

OBJECTIVE. To evaluate in patients with chronic renal failure (CRF) the effectiveness and the costs of sevelamer, a cationic polymer calcium- and aluminum-free, that is a new gastrointestinal phosphate binder.

Methods. Literature review and critical appraisal of six clinical trials about the effectiveness and two economic studies of sevelamer in CRF patients.

RESULTS. Sevelamer is an effective phosphate binder (used in a mean daily dose of $3.5 \mathrm{~g}$ three timesper day with meals) and with similar effect as that obtained with calcium salts, without the adverse manifestations of the latter (elevation of calcium x phosphorus product, hypercalcemia, vascular and cardiac calcifications, etc.). Moreover, sevelamer reduced serum LDL cholesterol in around $30 \%$. Despite the greater direct costs of sevelamer compared with calcium salts, the total costs may be lower due to the reduction of costs with clinical complications and hospitalizations.

Conclusions. Sevelamer has important therapeutic value in CRF patients with hyperphosphatemia. Economic analyses should be performed in our setting to define the cost-effectiveness relationship of sevelamer. [Rev Assoc Med Bras 2003; 49(I): 103-8]

KeYwords: Sevelamer. Hyperphosphatemia. Chronic Renal Failure, Secondary Hyperparathyroidism.

Este trabalho foi realizado com o apoio da Genzyme do Brasil

\section{REFERÊNCIAS}

I. Delmez JA, Slatopolsky E. Hyperphosphatemia: its consequences and treatment in patients with chronic renal disease. Am J Kidney Dis 1992; 19:303-17.

2. Slatopolsky E, Bricker NS. The role of phosphorus restriction in the prevention of secondary hyperparathyroidism in chronic renal diseases. Kidney Int 1993; 4: I II-5.

3. Slatopolsky E, Lopez-Hilker Delmez J, Dusso A, Brown A, Martin KJ. The parathyroid-calcitriol axis in health and chronic renal failure. Kidney Int I 986; 29(Suppl):S4 I-S7.

4. Piraino B, Chen T, Cooperstein L, Segre G, PuschettJ. Fractures and vertebral bone mineral density in patients with renal osteodystrophy. Clin Nephrol 1 988; 30:57-62.
5. Rao DS, Shih MS, Mohini R. Effect of serum parathyroid hormone and bone marrow fibrosis on the response to erythropoetin in uremia. N Engl J Med 1993; 328: I 7 I-5.

6. Massry SG, Goldstein DA. Role of parathyroid hormone in uremic toxicity. Kidney Int 1988;8 (Suppl):S39-S42.

7. Block GA, Hulbert-Shearon TE, Levin NW, Port FK. Association of serum phosphorus and calcium $x$ phosphate product with mortality risk in chronic hemodialysis patients: a national study. Am J Kidney Dis 1998;31:607- 17.

8. U.S. Renal Data System. USRDS 2000 Annual Data Report. National Institutes of Health, National Institutes of Diabetes and Digestive and Kidney Diseases. Bethesda; 2000.

9. Sesso R. Inquérito epidemiológico em unidades de diálise no Brasil. J Bras Nefrol 2000; 22(Supl 2):23-6.

10. Mailloux LU, Haley WE. Hypertension in the ESRD patient: pathophysiology, therapy, outcomes, and future directions. Am J Kidney Dis 1998; 32:705-17.

II. DeFronzo RA. Pathogenesis of glucose intolerance in uremia. Metabolism 1978; 27: 1866-80.

12. Avram MM, Goldwasser P, Burrell DE, Artignani A, Fein PA, Mittman N. The uremic dyslipidemia: a cross sectional and longitudinal study. Am J Kidney Dis 1992; 20:324-35.

13. Bostom AG, Shemin D, Lapane KL, Sutherland P, Nadeau MR, Wilson PW et al. Hyperhomocysteinemia, hyperfibrinogenemia, and lipoprotein (a) excess in maintenance dialysis patients: a matched case-control study. Atherosclerosis 1996; I 25:9|-101.

14. Block GA. Prevalence and clinical consequences of elevated $\mathrm{Ca} \times \mathrm{P}$ product in hemodialysis patients. Clin Nephrol 2000; 54:3 I 8-24.

15. Raggi P. Detection and quantification of cardiovascular calcifications with electron beam tomography to estimate risk in hemodialysis patients. Clin Nephrol 2000; 54:325-33.

16. Alfrey AC. Aluminium toxicity in patients with chronic renal failure. Ther Drug Monit 1993; 15:593-7.

17. Drueke TB, Lacour B, Towan M, Jacquel JP, Plachot JJ, Cournot-Witmer G, et al. Effect of aluminum on hematopoesis. Kidney Int I986; I8 (Suppl):S45-S8.

18. Arieff Al. Aluminum and the pathogenesis of dialysis encephalopathy. Am J Kidney Dis 1985; 6:3 |7-21.

19. Ramirez JA, Emmett M, White MG, Fathi N, Santa Ana CA, et al. The absorption of dietary phosphorus and calcium in hemodialysis patients. Kidney Int 1986; 30:753-9.

20. Spershneider H, Gunther K, Marzoll I, Kirchner E, Stein G. Calcium carbonate $\left(\mathrm{CaCO}_{3}\right)$ : an efficient and safe phosphate binder in hemodialysis patients? A 3-year study. Nephrol Dial Transplant 1993; 8:530-4

21. CookJD, DassenkoSA, Whittaker P. Calcium supplementation: effect on iron absorption. Am J Clin Nutr 1991; 53: 106-II.

22. Schwarz U, Buzello M, Ritz E, Stein G, Raabe 
G, Wiest G, et al. Morphology of coronary atherosclerotic lesions in patients with endstage renal failure. Nephrol Dial Transplant 2000; 15:218-23.

23. Goodman WG, Goldin J, Kuizon BD, Yoon C, Gales B, Sider D, et al. Coronary-artery calcification in young adults with end-stage renal disease who are undergoing dialysis. NEngIJ Med 2000; 342: 1478-83.

24. Ribeiro S, Ramos A, Brandao A, Rebelo JR, Guerra A, Resina C, et al. Cardiac valve calcification in haemodialysis patients: role of calcium-phosphate metabolism. Nephrol Dial Transplant 1998; 13:2037-40.

25. Hsu $\mathrm{CH}$. Are we mismanaging calcium and phosphate metabolism in chronic renal failure? Am J Kidney Dis 1997; 29:64 I-9

26. Guérin AP, London GM, Marchais SJ, Metivier F. Arterial stiffening and vascular calcifications in end-stage renal disease. Nephrol Dial Transplant 2000; 15:1014-21.

27. Burke SK, Slatopolsky EA, Goldberg DI. Renagel, a novel calcium- and aluminium-free phosphate binder, inhibits phosphate absorption in normal volunteers. Nephrol Dial Transplant 1997; I 2: 1640-4

28. Chertow GM, Burke SK, Lazarus JM, Stenzel $\mathrm{KH}$, Wombolt D, Goldberg D, et al. Poly [allylamine hydrochloride] (Renagel): A noncalcemic phosphate binder for the treatment of hyperphosphatemia in chronic renal failure. Am J Kidney Dis 1997; 29:66-7I.

29. Goldberg DI, Dillon MA, Slatopolsky EA, Garrett B, GrayJR, Marbury T, et al. Effect of Sevelamer, a non-aborbed, calcium-and aluminium-free phosphate binder, on serum phosphorus, calcium, and intact parathyroid hormone in end-stage renal disease patients. Nephrol Dial Transplant 1998; 13:2303-I0.

30. Chertow GM, Dillon M, Burke SK, Steg M, Bleyer AJ, Garrett BN, et al. A randomized trial of sevelamer hydrochloride (Renagel) with and without supplemental calcium. Clin Nephrol 1999; 51:18-26.

3I. Bleyer AJ, Burke SK, Dillon M, Garrett B, Kant $K S$, Lynch D, et al. A comparison of the calcium-free phosphate binder sevelamer hydrocloride with calcium acetate in the treatment of hyperphosphatemia in hemodialysis patients. Am J Kidney Dis 1999; 33:694701.

32. Slatopolsky EA, Burke SK, Dillon MA, The Sevelamer Study Group. Sevelamer, a nonabsorbed calcium- and aluminium-free phosphate binder, lowers serum phosphorus and parathyroid hormone. Kidney Int 1999; 55:299-307.

33. Chertow GM, Burke SK, Dillon MA, Slatopolsky E, The Sevelamer Study Group. Longterm effects of sevelamer hydrochloride on the calcium-phosphate product and lipid profile of haemodialysis patients. Nephrol Dial Transplant I999; I 4:2907-I4.

34. Bommer J, Strohbeck E, GoerichJ, Bahner M, Zuna I. Arteriosclerosis in dialysis patients. Int J Artif Organs 1996; 19:638-44

35. Nakagawa K. A study of aortic calcification in uremia. Jpn J Nephrol 1997; 39: I 35-43.

36. Raggi P, Burke SK, Dillon MA, Amin N, Bommer J, Chertow GM. Sevelamer attenuates the progression of coronary and aortic calcification compared with calcium-based phosphate binders. J Am Soc Nephrol 200I; I2:abstract I 232 .
37. Lowry EG, Lew NL. Death risk in hemodialysis patients: The predictive value of commonly measured variables and an evaluation of death rate differences between facilities. Am J Kidney Dis 1990; 15:458-82.

38. Tschope W, Koch M, Thomas B, Ritz E, The German Study Group Diabetes and Uremia. Serum lipids predict cardiac death in diabetic patients in maintenance hemodialysis. Nephron 1993; 64:354-8.

39. Drueke TB. A clinical approach to the uremic patient with extraskeletal calcification. Nephrol Dial Transplant 1996; I I:37-42.

40. Burke SK, Amin AS, Incerti C, Plone MA, Lee $J W$. Sevelamer hydrochloride $\left(\right.$ Renagel $\left.{ }^{R}\right)$, a phosphate-binding polymer, does not alter the pharmacokinetics of two commonly used antihypertensives in health volunteers. J Clin Pharmacol 200 I; 4 I: 199-205.

4I. Collins AJ, Peter WL, Dalleska FW, Ebben JP, Ma JZ. Hospitalization risks between Renagel phosphate binder treated and nonRenagel treated patients. Clin Nephrol 2000; 54:334-4I.

42. Brophy DF, Wallace JF, Kennedy DT, Gehr TWB, Holdford DA. Cost-effectiveness of sevelamer versus calcium carbonate plus atorvastatin to reduce LDL in patients with chronic renal insufficiency with dyslipidemia and hyperphosphatemia. Pharmacotherapy 2000; 20:950-7.

Artigo recebido: 12//2/2001

Aceito para publicação: 16/0I/2002 